\title{
39 CROSSING DISCIPLINARY BOUNDARIES: Reflections on Information Systems Research in Health Care and the State of Information Systems
}

\author{
Nicholas Barber \\ School of Pharmacy \\ University of London \\ Patricia Flatley Brennan \\ University of Wisconsin \\ Mike Chiasson \\ University of Calgary \\ Tony Cornford \\ London School of Economics and Political Science \\ Elizabeth Davidson \\ University of Hawaii, Manoa \\ Bonnie Kaplan \\ Yale University \\ Ela Klecuń \\ London School of Economics and Political Science
}

The Information Systems field has a long-standing research interest in the economic, social, and organizational implications of information technology - issues considered critically important in health care. Both IS and medical informatics are based in professional schools and draw heavily from studies of practice. To some researchers, it seems natural that the two fields would work closely together, drawing on and contributing to each other. Some think that IS research might benefit from testing, 
extending, or developing new theory in such a distinct organizational and institutional context. Others boldly advocate IS as a reference discipline for studying information systems in health care and call for stronger cross-referencing of IS research in the medical literature. If the IS field is ready to diffuse its knowledge into health care, what would be the terms and priority, given its usual business focus and business school base?

While IS research might be expected to flourish in health care, influencing policy and shaping practice, the reality is more sanguine. Each panel participant has experience crossing disciplinary boundaries to research information systems in health care. By examining similarities and differences between IS and medical informatics, we will offer some insight into the current state of IS as we assess methodological, institutional, and practical issues we face in our research. Comparing the two disciplines provides a provocative focus for surfacing assumptions about how IS research should be done and disseminated, for judging how well the objectives of the 1984 IFIP 8.2 conference call have been met, and for reflecting on the status of the so-called methods wars.

We aim to stimulate discussion by contrasting viewpoints on the following issues.

- How well has IS met the goals of the 1984 IFIP 8.2 conference, particularly in the field of health care?

- Can IS serve as a reference discipline for medical informatics? Should it?

- What can methodological differences in IS and medical informatics tell us about the IS methods wars?

- Medical researchers pay careful attention to ethical issues in constructing and undertaking research. What ethical considerations might be incorporated into IS research based on experience in clinical settings?

- What is generalizable or transferrable knowledge in IS? What might it look like? Have we achieved it? Should we try harder?

- Is it possible to provide theoretical bridges between some generalized IS theory and context-specific knowledge in health care so as to build and act upon multidisciplinary knowledge?

- What recommendations for cross-disciplinary work in health care (and other domains) can we provide?

- Do publication patterns and practices in each discipline reflect or drive significant or subtle differences in approaches to research and publication? 